Even at exploratory operation there may be difficulties. The affected area of the liver may be soft on palpation, but the liver may appear normal, when a large-bore needle must be passed into several areas to try to locate pus. In these circumstances preoperative real-time ultrasonography using a hand-held probe may be invaluable. ${ }^{13}$

When a unilocular abscess is found the cavity should be cleared, a large tube drain inserted, and treatment with appropriate antibiotics (such as gentamicin, metronidazole, and ampicillin) started immediately. Surgical drainage is undoubtedly inadequate alone. ${ }^{4}$ The drain should be left in place until there is sinographic evidence of obliteration of the cavity, and antibiotics should be continued for several weeks. ${ }^{11}$ With such a regimen $90 \%$ of patients with solitary abscesses will survive-an improvement on results from the preantibiotic era. ${ }^{4}$ In patients with multicentric abscesses the prognosis remains poor owing to the difficulty of obtaining satisfactory surgical drainage; fewer than a fifth of such patients will survive. ${ }^{134}$

${ }^{1}$ Heymann AD. Clinical aspects of grave pyogenic abscesses of the liver. Surg Gynecol Obstet 1979;149:209-13.

2 Silver S, Weinstein A, Cooperman A. Changes in the pathogenesis and detection of intrahepatic abscess. Am $\mathcal{f}$ Surg 1979;137:608-10.

3 Brodine WN, Schwartz SI. Pyogenic hepatic abscess. NY State 7 Med 1973;73:1657-61.

- Pitt HA, Zuidema GD. Factors influencing mortality in the treatment of pyogenic hepatic abscess. Surg Gynecol Obstet 1975;140:228-34.

5 Sherman JD, Robbins SL. Changing trends in the casuistics of hepatic abscess. $A m \mathcal{F}$ Med 1960;28:943-50.

- Rubin RH, Swartz MN, Malt R. Hepatic abscess: changes in clinical, bacteriologic and therapeutic aspects. Am $\mathcal{F}$ Med 1974;57:601-10.

7 Ochsner A, DeBakey M, Murray S. Pyogenic abscess of the liver. $\mathrm{Am} \mathcal{F}$ Surg 1938;11:292-319.

8 Palmer ED. The changing manifestations of pyogenic liver abscess. fAMA 1975;231:192.

- Young AE. The clinical presentation of pyogenic liver abscess. Br $\mathcal{F}$ Surg $1976 ; 63: 216-9$.

10 Schraibman IG. Non-parasitic liver abscess. Br $\mathcal{f}$ Surg 1974;61:709-12.

11 Butler TJ, McCarthy CF. Pyogenic liver abscess. Gut 1969;10:389-99.

12 Lawson TL. Hepatic abscess-ultrasound as an aid to diagnosis. Am $\mathcal{f}$ Dig Dis 1977;22:33-7.

${ }^{13}$ Lytton B, Cook J. Intraoperative ultrasound. In: Resnick MI, Saunders RC, eds. Ultrasound in urology. Baltimore: Williams and Wilkins, 1979:340-51.

\section{Is malignant diverticulitis a true bill?}

A recent report ${ }^{1}$ from California described 17 patients with a new clinical entity labelled "malignant" diverticulitis and characterised by extensive inflammation of the sigmoid and rectosigmoid, affecting all pelvic structures and often extending below the peritoneal reflection. Both obstruction and perforation were common, and many of the patients developed fistulas into adjacent viscera and on to the skin. Despite treatment with antibiotics, diversion of the faecal stream by colostomy, and other measures, a high proportion had postoperative complications and three died. The term "malignant" was applied because the diverticulitis behaved differently from ordinary diverticulitis in its progression and severity; there was no implication of neoplastic change. Nine out of 12 patients who had a colonic resection developed anastomotic leaks with ensuing sepsis and fistulas-a frightening complication rate by any standards, and one suggesting that there must have been some underlying cause for such alarming results.
Diverticula are never present in the rectum, ${ }^{2}$ and diverticulitis rarely affects more than the sigmoid colon. An inflammatory lesion of unusual extent, as in this so-called "malignant" diverticulitis, suggests some additional disease process. That was the case in the series described by Schmidt and his colleagues ${ }^{3}$ from St Mark's Hospital, London, of 26 patients with associated diverticulitis and Crohn's disease. Nine of their patients with a preoperative diagnosis of diverticulitis were subsequently shown to have coincidental Crohn's disease, and six developed postoperative complications of anastomotic leak, fistula formation, and sepsisalmost identical with those of "malignant" diverticulitis. In comparison, of 50 patients operated on at St Mark's Hospital for diverticulitis alone, only one developed a postoperative complication, an anal fistula.

In both the London and the California series the inflammation was intramural, severe, and extensive-a pattern more characteristic of Crohn's disease than diverticulitis alone, where the inflammation is mainly pericolic and extramural. Morgenstern et $a l^{1}$ admit the clinical similarity of "malignant" diverticulitis and granulomatous colitis but the pathological findings are, they say, consistent with severe diverticulitis. Nevertheless, the radiographic evidence of long intramural sinus tracts and the development of fistulas in 12 of their 17 patients are further evidence in favour of Crohn's disease rather than diverticulitis alone.

No mention is made in the report of the sigmoidoscopic findings, which could have been an important clue in the diagnosis of possible associated Crohn's disease. Anal lesions, mucosal granularity, pus, and ulceration suggest inflammatory bowel disease. A rectal biopsy is mandatory in patients with combined disease as there is a high incidence of abnormality. Morgenstern et al emphasise the absence of mucosal changes in their patients, though naked eye appearances alone do not entirely rule out the diagnosis of Crohn's disease.

Nor is anything said of the specific antibiotics used or of the culture of organisms found in the bowel lumen or in abscesses. Gram-negative anaerobes may have been responsible for the extensive inflammatory process that occurred in these 17 patients.

Granulomatous disease is known to coexist with diverticulitis in some patients and especially in the sigmoid colon in elderly women; and granulomatous colitis may masquerade as acute diverticulitis. ${ }^{4}$ Generally the distinction presents little diagnostic difficulty; but some patients may pose problems, especially after surgery for diverticulitis, when postoperative complications may be due to failure to recognise granulomatous disease. These patients are more likely to benefit from treatment with corticosteroids than from radical surgery.

The introduction of a new concept of "malignant" diverticulitis seems premature on the evidence so far available. When severe and progressive diverticulitis occurs in elderly patients with a high incidence of postoperative complications, extensive intramural inflammation, and the long sinus tracts, the doctor should assume that Crohn's disease is also present until the contrary is clearly proved.

1 Morgenstern L, Weiner R, Michel SL. "Malignant" diverticulitis a clinical entity. Arch Surg 1979;114:1112-6.

${ }^{2}$ Morson BC, Dawson IPM. Gastrointestinal pathology. Oxford: Blackwell, $1974: 499$.

s Schmidt GT, Lennard-Jones JE, Morson BC, Young AC. Crohn's disease of the colon and its distinction from diverticulitis. Gut 1968; 9:7-16.

4 Hoffman WA, Rosenberg MA. Granulomatous colitis in the elderly. Am $\mathcal{F}$ Gastroenterol 1972;58:508-18. 